\title{
Clinical outcomes and effects of treatment in older patients with idiopathic membranous nephropathy
}

Yaeni Kim ${ }^{1,2}$, Hye Eun Yoon ${ }^{1,3}$, Byung Ha Chung ${ }^{1,2}$, Bum Soon Choi ${ }^{1,2}$, Cheol Whee Park ${ }^{1,2}$, Chul Woo Yang ${ }^{1,2}$, Yong-Soo Kim ${ }^{1,2}$, Yu Ah Hong ${ }^{1,4}$, Suk Young Kim ${ }^{1,4}$, Yoon-Kyung Chang ${ }^{1,4}$, and Hyeon Seok Hwang ${ }^{5}$

${ }^{1}$ Division of Nephrology, Department of Internal Medicine, College of Medicine, The Catholic University of Korea, Seoul; ${ }^{2}$ Division of Nephrology, Department of Internal Medicine, College of Medicine, Seoul St. Mary's Hospital, The Catholic University of Korea, Seoul; ${ }^{3}$ Division of Nephrology, Department of Internal Medicine, College of Medicine, Incheon St. Mary's Hospital, The Catholic University of Korea, Incheon; ${ }^{4}$ Division of Nephrology, Department of Internal Medicine, College of Medicine, Daejeon St. Mary's Hospital, The Catholic University of Korea, Daejeon; ${ }^{5}$ Division of Nephrology, Department of Internal Medicine, Kyung Hee University Medical Center, Seoul, Korea

Received: April 12, 2018

Revised: June 12, 2018

Accepted: July 26, 2018

\section{Correspondence to}

Hyeon Seok Hwang, M.D.

Division of Nephrology, Department of Internal Medicine, Kyung Hee University Medical

Center, 23 Kyungheedae-ro,

Dongdaemun-gu, Seoul 02447,

Korea

Tel: $+82-32-280-5882$

Fax: $+82-32-280-5188$

E-mail: hwanghs@catholic.ac.kr
Background/Aims: Membranous nephropathy (MN) is the most common primary glomerular disease diagnosed in older patients. Few reports describe the clinical outcomes in older patients with idiopathic MN.

Methods: The outcomes of 135 patients with histologically proven MN were analyzed. 'Older' was defined as 60 years of age or older at the time of the renal biopsy. The rates of complete remission (CR), progression to end-stage renal disease (ESRD) and infection were compared between older and younger patients.

Results: The cumulative event rate for achieving CR was inferior $(p=0.012)$ and that for requiring renal replacement was higher $(p=0.015)$ in older patients, and they had a greater risk of infection $(p=0.005)$. Older age was a significant predictor of a lower rate of CR (adjusted odds ratio [OR], 0.51; 95\% confidence interval [CI], 0.26 to 0.98 ), and was a robust predictor of infection (adjusted OR, 5.27; 95\% CI, 1.31 to 21.20). Conservative treatment was associated with a lower remission rate ( $p$ $=0.036)$ and corticosteroid treatment was less effective in achieving $\mathrm{CR}(p=0.014)$, in preventing progression to $\operatorname{ESRD}(p=0.013)$ and in reducing infection $(p=0.033)$ in older patients. Cyclosporine treatment had similar clinical outcomes with regard to CR, ESRD progression, and infection in older patients.

Conclusions: Older age was independently associated with inferior rates of CR and greater risk of infection. Treatment modalities affected the outcomes of older patients differently in that cyclosporine treatment is predicted to be more useful than corticosteroids.

Keywords: Aged; Glomerulonephritis, membranous; Prognosis

\section{INTRODUCTION}

The number of older patients presenting with glomerular disease is growing with the increasing aver- age lifespan [1]. Membranous nephropathy $(\mathrm{MN})$ is the most common diagnosis of primary glomerular disease in older patients with a nephrotic syndrome and has a prevalence higher than that in younger adults [2]. Most 
MN in older patients has an idiopathic cause and the accuracy of diagnosis has been improved with the increasing trend towards performing renal biopsies [3]. Therefore, in an aging population, idiopathic $\mathrm{MN}$ is a major component of kidney disease in older patients. The associated morbidity and mortality has attracted attention to the clinical outcomes of $\mathrm{MN}$ in this population [4].

The natural history of the disease is often benign; those with non-nephrotic-range proteinuria invariably presents good prognosis in that they are responsive to symptomatic management including angiotensin receptor blockers (ARBs) and diuretics, thereby achieving spontaneous complete remission (CR) $[5,6]$. However, the $25 \%$ to $50 \%$ of patients presenting with heavy, persistent proteinuria, progressive renal functional deterioration, and unfavorable histological alterations are associated with poor clinical outcomes and may reach end-stage renal disease (ESRD) $[7,8]$. In these patients, the use of immunosuppressive agents may be justified to salvage failing kidneys and even for patient survival. However, little information is available about their outcomes because physicians are reluctant to use an aggressive treatment that may compromise the residual reservoir of already aged kidneys [9-11]. Therefore, information about the clinical outcomes of older patients compared with those in younger adults is vague and appropriate treatment modalities need to be determined and targeted to this aging population.

This study aimed to investigate the clinical characteristics of idiopathic MN in older patients. We explored the influence of older age on the clinical course and treatment responses of idiopathic $\mathrm{MN}$ in comparison with those in younger patients. In addition, we compared the complication rates associated with different treatment modalities in older and younger patients.

\section{METHODS}

\section{Study population}

We included patients with idiopathic MN who were treated at two medical centers of the Catholic University of Korea (Seoul and Daejeon St. Mary's Hospitals) from September 1998 to August 2016. Patients with histologically proven $\mathrm{MN}$ were screened and those with potential secondary causative factors were excluded by: clinical history with regard to possible drug use; serologic tests for both infectious and connective tissue diseases including serum autoantibodies, such as antinuclear antibody, anti-double-stranded DNA antibody, anti-neutrophil cytoplasmic antibody, and rheumatoid factor and complement; and screening for hepatitis B and hepatitis $\mathrm{C}$ viruses. Patients who had associated malignancies at presentation were also excluded from the study. Thorough clinical examination and appropriate laboratory screening excluded those with secondary causes for MN, leaving 135 patients diagnosed with idiopathic MN. We classified the study participants into 'older' and 'younger' groups, where 'older' was defined as 60 years of age or older at the time of the renal biopsy $[9,10]$.

\section{Data collection and definitions}

Data for age, sex, laboratory test results, treatment modalities, clinical responses to treatment, progression to ESRD, occurrence of infection, and patient survival were collected. Body mass index was calculated as the ratio of weight in kilograms divided by the square of the height in meters. Estimated glomerular filtration rate (eGFR) was determined from serum creatinine concentration, age, sex, and race using the abbreviated Modification of Diet in Renal Disease (MDRD) formula [12]. Protein concentration in a 24-hour urine sample or the protein-creatinine ratio of spot urine was used to estimate the amount of proteinuria. Treatment modalities were classified into four groups: conservative treatment, corticosteroids alone (at least $50 \mathrm{mg}$ prednisone/day), cyclophosphamide with or without steroids (less than $15 \mathrm{mg}$ prednisone/day), or cyclosporine with or without steroids (less than $15 \mathrm{mg}$ prednisone/day).

Renal biopsy specimens were examined by light microscopy, immunofluorescence microscopy, and electron microscopy. Sections $(2 \mu \mathrm{m})$ stained with hematoxylin and eosin, Masson's trichrome, periodic acid-Schiff, and periodic acid-silver methenamine were examined by light microscopy. The number of global or segmental sclerotic glomeruli is presented as the percentage of the total number of glomeruli in the biopsy specimen. The degree of tubular atrophy was graded from o to 3 (mild, moderate, and severe). Interstitial fibrosis was graded as follows: grade o, none; grade $1,<25 \%$; grade $2,26 \%$ to $50 \%$; and grade $3,>50 \%$. Cryosections $(4 \mu \mathrm{m})$ were stained with fluorescein isothiocyanate-conjugated rab- 
bit anti-human immunoglobulin $\mathrm{G}(\operatorname{IgG})$, IgA, IgM, к and $\lambda$ light chains, and complement $3\left(\mathrm{C}_{3}\right), \mathrm{C}_{4}$, and $\mathrm{C} 1 \mathrm{q}$.

\section{Outcome measures}

The primary endpoint was treatment response, which was assessed as CR, partial remission (PR), or no response (NR). CR was defined as urinary protein excretion less than $300 \mathrm{mg} /$ day and stable serum creatinine level. PR was defined as urine protein excretion less than 3.5 $\mathrm{g} /$ day or less than $50 \%$ of baseline proteinuria with stable serum creatinine level. NR was defined when urinary protein excretion remained at over $50 \%$ of the baseline value or serum creatinine level progressively increased $[4,13,14]$. The secondary endpoints were progression to ESRD, the occurrence of infection and patient death. Development of infection was defined as such that required hospitalization. ESRD was considered a prerequisite for renal replacement therapies such as maintenance dialysis and kidney transplantation.

\section{Statistical analysis}

Variables are presented as the mean $\pm \mathrm{SD}$ or as frequencies (percentage). Continuous variables were compared using Student's $t$ test, and categorical variables were compared using a chi-square test or Fisher's exact test. To test for an independent association of older age and the occurrence of endpoints, a Cox proportional hazards model was used. Such variables that were deemed clinically significant (i.e., the presence of hypertension, proteinuria, treatment modality, and hemoglobin level) were entered into multivariate Cox regression analyses along with variables of $p$ value less than 0.1 in univariate analyses. The cumulative event rates for the prespecified endpoint were estimated by the Kaplan-Meier method and compared using log-rank tests. A $p<0.05$ was considered significant. Statistical analysis was performed using SPSS software version 20.0 (IBM Corp., Armonk, NY, USA).

\section{Ethics approval and consent to participate}

The study was approved by the Institutional Review Board at College of Medicine, the Catholic University of Korea (XC15RIMIoo71K). Informed consent was waived due to the retrospective nature of the study.

\section{RESULTS}

\section{Baseline characteristics of older and younger pa- tients with idiopathic MN}

A total of 135 patients included 77 men (57\%) and 58 women $(43 \%)$. The mean age was $53.7 \pm 14.4$ years. Eighteen $(13 \%)$ and 41 patients (30\%) had diabetes and hypertension, respectively. The mean eGFR was $99.0 \pm 41.9 \mathrm{~mL} /$ $\mathrm{min} / 1.73 \mathrm{~m}^{2}$ and 19 patients (14.1\%) had eGFR less than $60 \mathrm{~mL} / \mathrm{min} / 1.73 \mathrm{~m}^{2}$. The amount of proteinuria ranged from 0.02 to $18.02 \mathrm{~g} /$ day and patients with nephrotic range proteinuria (>3 g/day) accounted for 103 (76\%). Of these patients, 48 (36\%) were treated conservatively with the use of ARBs, while 87 patients (64\%) were assigned to treatment with immunosuppressive agents.

A greater proportion of older patients than of younger patients had hypertension. Compared with younger patients, older patients presented with lower eGFR. There were no significant differences in serum albumin level, lipid profiles, and the amount of proteinuria. The proportion of those with nephrotic range proteinuria was more prevalent in the older group. The biopsy findings in older patients demonstrated unfavorable histologic changes (Table 1).

\section{Comparison of clinical outcomes between older and younger patients with idiopathic MN}

In the older patient group, 18 (33.3\%) achieved CR, 14 (25.9\%) had PR, and 22 (40.7\%) had NR. Younger patients had significantly more favorable responses to treatment than older patients did (CR 61.7\%, PR 11.1\%, NR 27.2\%, $p=0.004)$. The cumulative event rate for achieving $C R$ was significantly inferior in older patients compared with younger patients $(p=0.012$ ) (Fig. $1 \mathrm{~A})$. Compared to younger patients, older patients with nephrotic range proteinuria achieved less CR (30.4\% vs. $54.4 \%, p=0.013$ ) and those with non-nephrotic range proteinuria had more NR (50.0\% vs. $8.3 \%, p=0.026)$.

Two deaths (3.7\%) occurred in the older patient group ( $p=0.158$ vs. younger patients). The cumulative event rate for requiring renal replacement therapy was significantly higher in older patients $(p=0.015)$ (Fig. 1B). Older patients had a greater risk of infection and a significant difference was observed between the age groups in the development of infection ( $p=0.005$ ) (Fig. 1C). 
Table 1. Baseline characteristics of elderly and young patients with idiopathic membranous nephropathy

\begin{tabular}{|c|c|c|c|}
\hline Characteristic & Elderly patients $(\mathrm{n}=54)$ & Young patients $(\mathrm{n}=8 \mathrm{1})$ & $p$ value \\
\hline Age, yr & $67.2 \pm 5.6$ & $44.6 \pm 10.9$ & $<0.001$ \\
\hline Male sex & $33(61.1)$ & $44(55 \cdot 6)$ & 0.522 \\
\hline Body mass index, $\mathrm{kg} / \mathrm{m}^{2}$ & $24 \cdot 6 \pm 3 \cdot 5$ & $23 \cdot 9 \pm 3 \cdot 3$ & 0.278 \\
\hline Diabetes & $8(15.1)$ & $10(12.3)$ & 0.648 \\
\hline Hypertension & $28(52.8)$ & $13(16.0)$ & $<0.001$ \\
\hline Systolic blood pressure, $\mathrm{mmHg}$ & $130.5 \pm 18.9$ & $123.1 \pm 17.5$ & 0.022 \\
\hline Diastolic blood pressure, $\mathrm{mmHg}$ & $78.7 \pm 10.3$ & $75.8 \pm 11.0$ & 0.129 \\
\hline Follow-up duration, mon & $48.2 \pm 46.0$ & $63.0 \pm 49.1$ & 0.134 \\
\hline \multicolumn{4}{|l|}{ Laboratory findings } \\
\hline eGFR, $\mathrm{mL} / \mathrm{min} / 1.73 \mathrm{~m}^{2}$ & $80.6 \pm 38.6$ & $111.2 \pm 39.6$ & $<0.001$ \\
\hline Hemoglobin, g/dL & $12.3 \pm 2.0$ & $13.8 \pm 1.9$ & $<0.001$ \\
\hline Serum albumin, g/dL & $2.5 \pm 0.8$ & $2.8 \pm 0.8$ & 0.055 \\
\hline Total cholesterol, mg/dL & $294.4 \pm 95.6$ & $296.4 \pm 117.3$ & 0.919 \\
\hline Triglyceride, mg/dL & $209.8 \pm 135.6$ & $216.6 \pm 164.9$ & 0.802 \\
\hline Proteinuria, g/day & $6.4 \pm 3.4$ & $5 \cdot 7 \pm 4 \cdot 3$ & 0.323 \\
\hline$>3 \mathrm{~g} /$ day proteinuria, $\%$ & $46(85 \cdot 2)$ & $57(70.4)$ & 0.047 \\
\hline Range proteinuria (min-max) & $1.3-15 \cdot 5$ & $0.02-18.0$ & \\
\hline \multicolumn{4}{|l|}{ Biopsy findings } \\
\hline Global sclerosis, \% & $14 \cdot 5 \pm 14 \cdot 7$ & $7 \cdot 7 \pm 11.7$ & 0.005 \\
\hline Segmental sclerosis, \% & $5.6 \pm 9.7$ & $3.6 \pm 6.7$ & 0.218 \\
\hline Tubular atrophy grade & $1.4 \pm 0.7$ & $0.7 \pm 0.7$ & $<0.001$ \\
\hline Interstitial fibrosis grade & $1.4 \pm 0.7$ & $0.7 \pm 0.7$ & $<0.001$ \\
\hline \multicolumn{4}{|l|}{ Treatment } \\
\hline Conservative treatment & $18(33 \cdot 3)$ & $30(37.0)$ & 0.660 \\
\hline Corticosteroid & $18(33 \cdot 3)$ & $22(27.2)$ & 0.442 \\
\hline Cyclophosphamide & $6(11.1)$ & $5(6.2)$ & 0.347 \\
\hline Cyclosporine & $12(22.2)$ & $24(29.6)$ & 0.340 \\
\hline
\end{tabular}

Values are presented as mean \pm SD or number (\%).

eGFR, estimated glomerular filtration rate.

\section{Older age is an independent predictor of CR and infection}

Univariate Cox proportional hazard models showed that older age and male sex decreased the odds ratio (OR) for CR and an increased eGFR was positively associated with CR from proteinuria (Table 2). After adjusting for clinically relevant factors, older age and male sex remained as significant predictors of a reduced chance of CR from proteinuria. Both univariate and multivariate models revealed older age as a robust predictor of the development of infection. In univariate models, older patients had a significantly greater risk of progression to ESRD (OR, 10.91; 95\% confidence interval [CI], 2.11 to 56.36; $p=0.004)$, but this association was not significant in multivariate models (adjusted OR, 2.08; 95\% CI, 0.26 to $16.47 ; p=0.486$ ).

\section{Comparison of clinical outcomes in older and younger patients based on treatment modalities}

The effectiveness of different treatment strategies in older and younger patients was analyzed in terms of CR attainment, development of ESRD, and infection. Conservative treatment was associated with a significantly lower remission rate in older patients (Fig. 2A). 


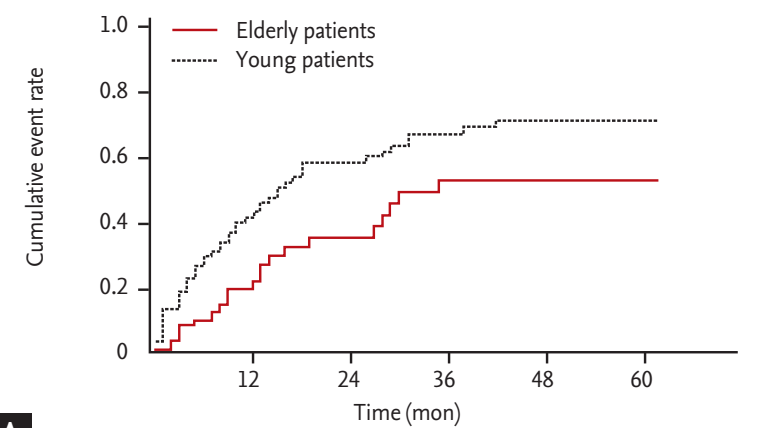

A

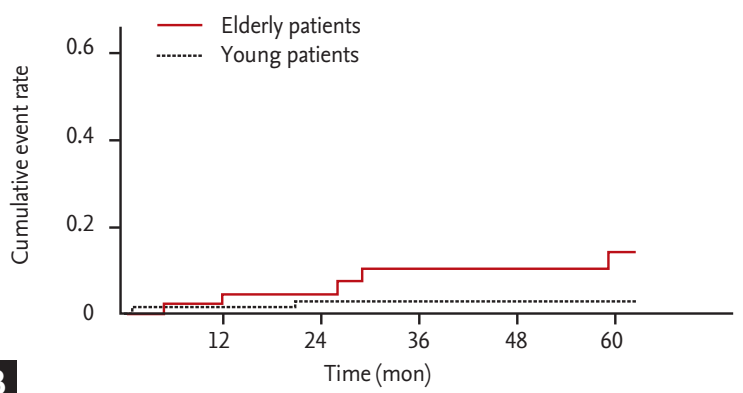

B

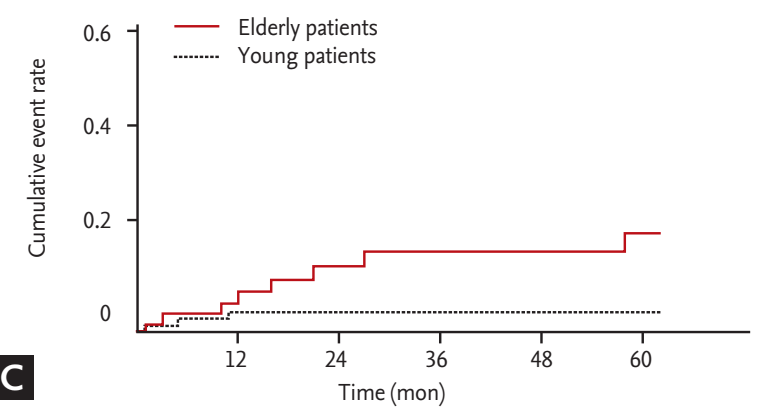

Figure 1. Cumulative event rates for (A) complete remission, (B) progression to end-stage renal disease (ESRD), and (C) infection in older and younger patients by month. The cumulative event rate for complete remission is significantly higher in younger patients compared with older patients $(p$ $<0.012$ ). Older patients were associated with poorer clinical outcomes in terms of significantly greater cumulative event rates for both progression to $\operatorname{ESRD}(p<0.015)$ and infection $(p$ $<0.005)$.

No significant differences were observed between the age groups in terms of renal progression and occurrence of infectious complications in patients managed with conservative treatment. Corticosteroid treatment was less effective in the older patient group $(n=18)$ in achieving CR and preventing progression to ESRD, and moreover was associated with a greater frequency of infectious complications (Fig. 2B) than in the younger patient group $(\mathrm{n}=22)$. Cyclosporine showed comparable effectiveness for achieving CR and in preventing renal functional deterioration and infectious complications between older and younger patients $(n=12$ and $n=24$, respectively) (Fig. 2C). The number of patients treated with cyclophosphamide was limited, but this treatment was associated with comparable rates of CR (50.0\% vs. $80.0 \%, p=0.545)$ and infectious complications $(16.7 \%$ vs. $0.0 \%, p=1.00)$ in older and younger patients $(n=6$ and $\mathrm{n}=5$, respectively).

\section{DISCUSSION}

Our study demonstrated that older patients with idiopathic MN had a lower CR rate, higher rates of progression to ESRD, and greater frequency of infectious complications than younger patients. Of the treatment modalities, conservative treatment was less effective in achieving CR in older patients and corticosteroid therapy had less clinical benefit. These findings suggest that older patients with idiopathic MN have an unfavorable clinical course compared with that of younger patients and that treatment modalities affect the outcomes of older patients differently from younger patients.

The commonest manifestation of idiopathic MN is nephrotic syndrome with preserved kidney function $[15,16]$. The clinical presentation of older patients is not so much different from that of younger adults in that heavy proteinuria with the presence of edema is the most prominent finding at the time of renal biopsy [17], which is consistent with the results of this study because both patients had heavy proteinuria and comparable levels of serum cholesterol and albumin. However, these older patients had a significantly lower eGFR and a higher prevalence of hypertension, with a greater proportion of glomerular sclerosis and more severe grades of tubular atrophy and interstitial fibrosis on renal biopsies. These findings suggest that older patients are predisposed to a higher risk of progression to ESRD than younger patients, despite the similar initial clinical presentation of idiopathic MN in the two groups.

In the present study, we found that older patients showed a lower rate of CR than younger patients did, and that age was independently associated with an inferior $\mathrm{CR}$ rate. In addition, a higher rate of progression to ESRD was observed in older patients, although the treat- 
Table 2. Univariate and multivariate analyses of predictors for complete remission and infection

\begin{tabular}{|c|c|c|c|c|}
\hline \multirow{2}{*}{ Variable } & \multicolumn{2}{|c|}{ Univariate analysis } & \multicolumn{2}{|c|}{ Multivariate analysis } \\
\hline & OR $(95 \% \mathrm{CI})$ & p value & $\mathrm{OR}(95 \% \mathrm{CI})$ & $p$ value \\
\hline \multicolumn{5}{|l|}{ Complete remission } \\
\hline Elderly & $0.53(0.32-0.88)$ & 0.014 & $0.51(0.26-0.98)$ & 0.045 \\
\hline Male sex & $0.46(0.29-0.73)$ & 0.001 & $0.51(0.30-0.88)$ & 0.016 \\
\hline Diabetes & $0.47(0.20-1.07)$ & 0.073 & $0.49(0.20-1.17)$ & 0.113 \\
\hline Hypertension & $0.73(0.43-1.23)$ & 0.236 & $1.15(0.60-2.19)$ & 0.684 \\
\hline Hemoglobin (per 1 g/dL increment) & $0.95(0.84-1.07)$ & 0.399 & $0.94(0.81-1.10)$ & 0.455 \\
\hline eGFR (per $10 \mathrm{~mL} / \mathrm{min} / 1.73 \mathrm{~m}^{2}$ increment) & $1.09(1.01-1.17)$ & 0.020 & $1.06(0.98-1.15)$ & 0.174 \\
\hline Proteinuria (per 1 g/day increment) & $0.96(0.90-1.03)$ & 0.251 & $0.99(0.93-1.06)$ & 0.843 \\
\hline \multicolumn{5}{|l|}{ Treatment (vs. conservative treatment) } \\
\hline Cyclophosphamide & $1.29(0.55-3.00)$ & 0.558 & $1.55(0.65-3.70)$ & 0.329 \\
\hline Corticosteroid & $1.19(0.67-2.09)$ & 0.557 & $1.01(0.56-1.83)$ & 0.979 \\
\hline Cyclosporine A & $0.90(0.48-1.67)$ & 0.737 & $0.81(0.42-1.58)$ & 0.535 \\
\hline \multicolumn{5}{|l|}{ Infection } \\
\hline Elderly & $5.29(1.45-19.27)$ & 0.012 & $5.27(1.31-21.20)$ & 0.019 \\
\hline Male sex & $0.59(0.20-1.75)$ & 0.338 & - & \\
\hline Diabetes & $0.50(0.07-3.85)$ & 0.506 & $0.48(0.06-3.85)$ & 0.487 \\
\hline Hypertension & $1.98(0.67-5.91)$ & 0.219 & - & \\
\hline Hemoglobin (per 1 g/dL increment) & $0.83(0.62-1.11)$ & 0.212 & $0.98(0.68-1.40)$ & 0.913 \\
\hline eGFR (per $10 \mathrm{~mL} / \mathrm{min} / 1.73 \mathrm{~m}^{2}$ increment) & $0.89(0.74-1.06)$ & 0.188 & $0.99(0.81-1.22)$ & 0.957 \\
\hline Proteinuria (per 1 g/day increment) & $1.02(0.89-1.16)$ & 0.773 & $1.05(0.89-1.25)$ & 0.556 \\
\hline \multicolumn{5}{|l|}{ Treatment (vs. conservative treatment) } \\
\hline Cyclophosphamide & $1.14(0.12-11.01)$ & 0.909 & $0.70(0.06-8.02)$ & 0.771 \\
\hline Corticosteroid & $2.59(0.67-10.03)$ & 0.167 & $2.29(0.54-9.61)$ & 0.260 \\
\hline Cyclosporine A & $0.98(0.16-5.86)$ & 0.980 & $1.06(0.16-7.10)$ & 0.956 \\
\hline
\end{tabular}

OR, odds ratio; CI, confidence interval; eGFR, estimated glomerular filtration rate.

ment modalities and the frequency of their use were similar to those of younger patients. These findings suggest that treatment response is significantly inferior in older patients than in younger patients. Contemplating on the reasons for poorer treatment response in the older group, we suggest that the higher proportion of those with nephrotic-range proteinuria at presentation may contribute to lower treatment response. Moreover, it is presumed that appropriate timing for the initiation of immunosuppressive agents may have been delayed in the older group (data not shown; $0.9 \pm 2.2$ months vs. $5.8 \pm 18.0$ months, $p=0.057$ ). These findings suggest that treatment strategy for dealing with older patients needs to be more active than current clinical practice.
Older patients demonstrated greater cumulative event rates for the development of infection. In addition, time to the occurrence of infectious complications was more extended than that of younger patients (data not shown; $34.0 \pm 36.4$ months vs. $5.7 \pm 5.0$ months, $p=0.038$ ). Among a total of 36 elderly patients who had taken immunosuppressant, eight developed infectious complications. Four of them developed infection while on drugs, and the other four showed signs of infection after finishing the intake of immunosuppressive agents. These results suggest that elderly patients were at higher risk for infection than younger patients and that cautious observation is necessary even after the patient have finished the prescribed dose of immunosuppressant. 

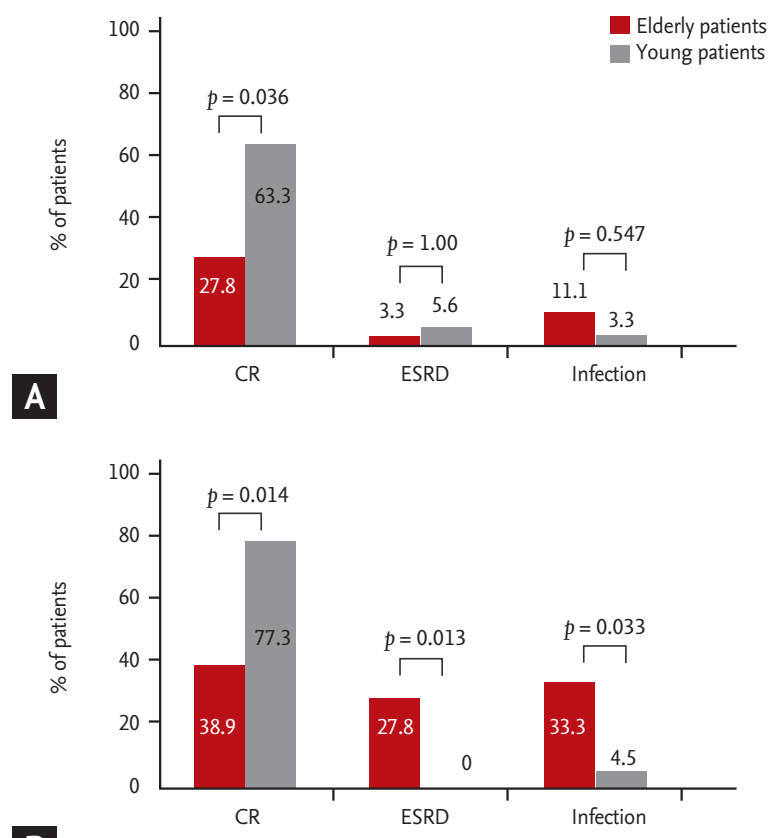

B

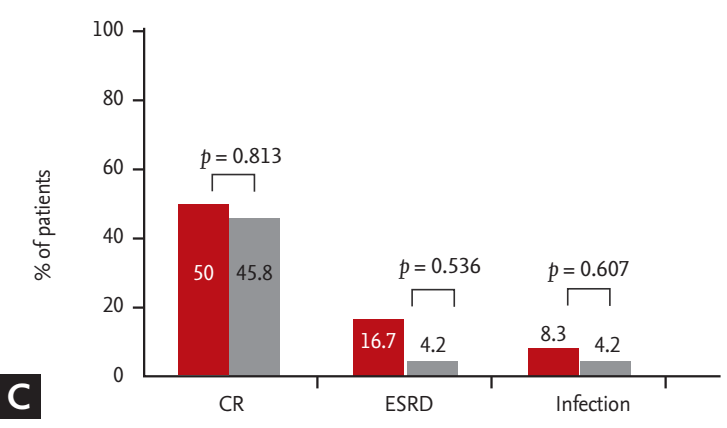

Figure 2. Clinical outcomes according to different treatment regimens in older and younger patients with idiopathic membranous nephropathy in terms of complete remission (CR) attainment, progression to end-stage renal disease (ESRD), and development of infectious complications: (A) conservative treatment, (B) corticosteroid, and (C) cyclosporine. Conservative treatment was associated with favorable renal outcomes in younger patients but not in older patients in terms of CR rate (60.5\% vs. $20 \%, p=0.044)$. The proportion of patients who progressed to ESRD and developed infection did not differ significantly between the two groups under conservative treatment. Corticosteroid treatment was associated with poorer clinical outcomes in older patients: a greater percentage of older patients progressed to ESRD and developed infectious complications $(27.8 \%$ vs. $0 \%, p=0.013$; $33.3 \%$ vs. $4.5 \%, p=0.033$, respectively). For renal parameters, corticosteroid treatment was associated with less CR attainment in older patients (44.4\% vs. $77.3 \%, p=0.003)$. Cyclosporine showed comparable rates of CR, progression to ESRD, and development of infection in the two groups.
About $50 \%$ of idiopathic MN patients with persistent high-grade proteinuria eventually progress to ESRD and aggressive therapy is usually initiated in this context [18]. The present study suggests that because older patients were associated with poorer renal outcomes with respect to increased event rates for renal replacement therapy, these patients should be eligible to receive aggressive treatment regimen to preserve renal function. However, our study also demonstrated that older patients are exposed to greater risks of infectious complications during treatment [13]. These findings suggest that an aggressive treatment strategy should be individualized in older patients to balance the risks and benefits of treatment.

The natural history of idiopathic MN in older patients is often benign, with some individuals eventually experiencing spontaneous remission of nephrotic syndrome [19]. Therefore, it is reasonable to prefer conservative treatment in older patients when we consider their higher potential risk of infectious complications. In this study, while symptomatic treatment resulted in comparable rates of infectious complications and ESRD progression in older and younger patients, CR was achieved in only $20 \%$ of older patients, which was significantly lower than the rate in younger patients. These findings suggest that conservative treatment was ineffective in achieving CR in older patients and that immunosuppressant should be considered as a treatment option in this context. We suggest that conservative treatment is effective in those with a high risk of infectious complications or with limited lifespan that reduces their risk of developing ESRD [20].

Several randomized trials have failed to demonstrate the efficacy of glucocorticoid monotherapy in the treatment of idiopathic MN, despite early encouraging studies involving patients of Asian ancestry that reported its potential benefit [21-23]. Our study demonstrated that corticosteroid treatment in older patients was associated with unfavorable clinical outcomes including lower CR rates and greater risks of progression to ESRD and development of infectious conditions compared with younger patients. These findings confirm that corticosteroid monotherapy has limited value as a treatment option in older patients and that it is associated with a higher complication rate $[15,24,25]$. In contrast, cyclosporine use showed comparable results in older and younger patients with respect to achievement of CR, 
progression to ESRD, and occurrence of infectious complications. On these grounds, we cautiously infer that cyclosporine may produce comparably favorable outcomes for renal aspects and therefore, it may be considered an alternative first-line treatment option for older people who are vulnerable to infections.

Our study has limitations. First, it was based on retrospective data analysis. Treatment modality was not standardized; rather it was based on an individual practitioner's decision depending on patients' clinical setting. Therefore, there are unmeasured biases in evaluating primary and secondary outcomes. Second, patients clearly were not randomized to immunosuppressive modalities, and potential confounders may exist. We adjusted for relevant key clinical parameters in the analyses, but there may be factors influencing treatment decisions that were included in our models. Therefore, prospective randomized controlled studies are required to compensate for these flaws. Third, we could not evaluate the effect of mycophenolate mofetil owing to insurance policy, and no patients received the cyclic therapy of corticosteroid and cyclophosphamide.

In conclusion, older age in patients with idiopathic $\mathrm{MN}$ was associated with an inferior clinical course and worse treatment responses. The achievement of $\mathrm{CR}$, progression to ESRD, and occurrence of infectious complications for each treatment regimen was significantly altered in older patients compared with younger patients. The use of corticosteroid therapy was associated with minimal clinical benefit, but cyclosporine treatment is predicted to have the potential to lead to a better prognosis. Therefore, our study provides valuable information that helps to improve the clinical outcomes in older patients with idiopathic MN.

\section{KEY MESSAGE}

1. Cumulative event rate for achieving complete remission was inferior and that for requiring dialysis, and infection was higher in older patients.

2. Old age per se was independently associated with inferior clinical outcomes in patients with idiopathic membranous nephropathy.

3. Cyclosporine treatment is predicted to be more useful than corticosteroids in older patients

\section{Conflict of interest}

No potential conflict of interest relevant to this article was reported.

\section{Acknowledgments}

This research was supported by the Basic Science Research Program through the National Research Foundation of Korea (NRF) funded by the Ministry of Education, Science and Technology (2015R1A1A1A05001599).

\section{REFERENCES}

1. Sumnu A, Gursu M, Ozturk S. Primary glomerular diseases in the elderly. World J Nephrol 2015;4:263-270.

2. Honkanen E, Tornroth T, Gronhagen-Riska C. Natural history, clinical course and morphological evolution of membranous nephropathy. Nephrol Dial Transplant 1992;7 Suppl 1:35-41.

3. Zhu P, Zhou FD, Zhao MH. The renal histopathology spectrum of elderly patients with kidney diseases: a study of 430 patients in a single Chinese center. Medicine (Baltimore) 2014;93:e226.

4. Pan $\mathrm{X}, \mathrm{Xu}$ J, Ren $\mathrm{H}$, et al. Changing spectrum of biopsy-proven primary glomerular diseases over the past 15 years: a single-center study in China. Contrib Nephrol 2013;181:22-30.

5. Hladunewich MA, Troyanov S, Calafati J, Cattran DC; Metropolitan Toronto Glomerulonephritis Registry. The natural history of the non-nephrotic membranous nephropathy patient. Clin J Am Soc Nephrol 2009;4:14171422.

6. Schieppati A, Mosconi L, Perna A, et al. Prognosis of untreated patients with idiopathic membranous nephropathy. N Engl J Med 1993;329:85-89.

7. Honkanen E. Survival in idiopathic membranous glomerulonephritis. Clin Nephrol 1986;25:122-128.

8. Murphy BF, Fairley KF, Kincaid-Smith PS. Idiopathic membranous glomerulonephritis: long-term follow-up in 139 cases. Clin Nephrol 1988;30:175-181.

9. Yoon HE, Shin MJ, Kim YS, et al. Clinical impact of renal biopsy on outcomes in elderly patients with nephrotic syndrome. Nephron Clin Pract 2011;117:c20-c27.

10. Davison AM, Johnston PA. Glomerulonephritis in the elderly. Nephrol Dial Transplant 1996;11 Suppl 9:34-37.

11. Moutzouris DA, Herlitz L, Appel GB, et al. Renal biopsy in 
the very elderly. Clin J Am Soc Nephrol 2009;4:1073-1082.

12. Matsuo S, Imai E, Horio M, et al. Revised equations for estimated GFR from serum creatinine in Japan. Am J Kidney Dis 2009;53:982-992.

13. Yamaguchi M, Ando M, Yamamoto R, et al. Patient age and the prognosis of idiopathic membranous nephropathy. PLoS One 2014;9:e110376.

14. Yoshimoto K, Yokoyama H, Wada T, et al. Pathologic findings of initial biopsies reflect the outcomes of membranous nephropathy. Kidney Int 2004;65:148-153.

15. Cameron JS. Nephrotic syndrome in the elderly. Semin Nephrol 1996;16:319-329.

16. Deegens JK, Wetzels JF. Membranous nephropathy in the older adult: epidemiology, diagnosis and management. Drugs Aging 2007;24:717-732.

17. Yokoyama H, Sugiyama H, Narita I, et al. Outcomes of primary nephrotic syndrome in elderly Japanese: retrospective analysis of the Japan Renal Biopsy Registry (J-RBR). Clin Exp Nephrol 2015;19:496-505.

18. O'Callaghan CA, Hicks J, Doll H, Sacks SH, Cameron JS. Characteristics and outcome of membranous nephropathy in older patients. Int Urol Nephrol 2002;33:157-165.
19. Zent R, Nagai R, Cattran DC. Idiopathic membranous nephropathy in the elderly: a comparative study. Am J Kidney Dis 1997;29:200-206.

20. du Buf-Vereijken PW, Branten AJ, Wetzels JF. Idiopathic membranous nephropathy: outline and rationale of a treatment strategy. Am J Kidney Dis 2005;46:1012-1029.

21. Ponticelli C, Glassock RJ. Glomerular diseases: membranous nephropathy: a modern view. Clin J Am Soc Nephrol 2014;9:609-616.

22. Collaborative Study of the Adult Idiopathic Nephrotic Syndrome. A controlled study of short-term prednisone treatment in adults with membranous nephropathy. $\mathrm{N}$ Engl J Med 1979;301:1301-1306.

23. Shiiki H, Saito T, Nishitani Y, et al. Prognosis and risk factors for idiopathic membranous nephropathy with nephrotic syndrome in Japan. Kidney Int 2004;65:1400-1407.

24. Abrass CK. Treatment of membranous nephropathy in the elderly. Semin Nephrol 2003;23:373-378.

25. Kalliakmani P, Koutroulia E, Sotsiou F, Vlachojannis JG, Goumenos DS. Benefit and cost from the long-term use of cyclosporine-A in idiopathic membranous nephropathy. Nephrology (Carlton) 2010;15:762-767. 